\title{
Tangent-Impulse Interception for a Hyperbolic Target
}

\author{
Dongzhe Wang, Gang Zhang, and Xibin Cao \\ Research Center of Satellite Technology, Harbin Institute of Technology, Harbin 150080, China \\ Correspondence should be addressed to Gang Zhang; zhanggang3110@163.com
}

Received 30 November 2013; Accepted 30 March 2014; Published 28 April 2014

Academic Editor: Antonio F. Bertachini A. Prado

Copyright (C) 2014 Dongzhe Wang et al. This is an open access article distributed under the Creative Commons Attribution License, which permits unrestricted use, distribution, and reproduction in any medium, provided the original work is properly cited.

\begin{abstract}
The two-body interception problem with an upper-bounded tangent impulse for the interceptor on an elliptic parking orbit to collide with a nonmaneuvering target on a hyperbolic orbit is studied. Firstly, four special initial true anomalies whose velocity vectors are parallel to either of the lines of asymptotes for the target hyperbolic orbit are obtained by using Newton-Raphson method. For different impulse points, the solution-existence ranges of the target true anomaly for any conic transfer are discussed in detail. Then, the time-of-flight equation is solved by the secant method for a single-variable piecewise function about the target true anomaly. Considering the sphere of influence of the Earth and the upper bound on the fuel, all feasible solutions are obtained for different impulse points. Finally, a numerical example is provided to apply the proposed technique for all feasible solutions and the global minimum-time solution with initial coasting time.
\end{abstract}

\section{Introduction}

The earth-crossing comets, asteroids, space debris, and other spacecraft are potentially hazardous objects (PHOs) since they may closely encounter with the Earth. Usually, an active spacecraft is required to intercept, impact, or deflect the $\mathrm{PHO}$ [1-4]. The impact and deflection mission is mainly used when the PHO is beyond the sphere of influence of the Earth. If this mission is failed, the $\mathrm{PHO}$ will be on a hyperbolic trajectory during the Earth flyby; thus the interception problem from a parking orbit for a hyperbolic target needs to be solved.

Assume the nonmaneuvering target is subject only to a Newtonian gravitational attraction and the Lambert approach is the most fundamental and the simplest method to accomplish the two-body interception problem with impulse maneuvers [5-7]. If the impulse point and the interception point are both assigned, the time of flight (TOF) for the target can be obtained by solving Kepler's equation of hyperbolic orbit, and then the interception problem is equivalent to Lambert's problem [8-11]. The required velocity vector of the transfer trajectory at the impulse point can be obtained with the Lambert approach. It should be notified that the transfer trajectory can be elliptic, parabolic, and hyperbolic.
When the initial coasting time is considered, the impulse point may be not at the initial time, then interception optimization problems to save time and fuel are studied by many researchers [12-14].

However, no constraints are imposed on the impulse direction for the Lambert approach when both impulse and interception points are assigned. If two coplanar orbits are tangent and the velocity vectors at their common point are in the same direction, then the task of nulling the relative velocity will be simple [8]. The cotangent transfer indicates that the transfer orbit is tangent to both the initial orbit and the final orbit. The classic Hohmann transfer [15] is a twoimpulse cotangent transfer between two coplanar circular orbits, and it is the minimum-energy one among all the twoimpulse transfers. This result is also satisfied for two coplanar elliptic orbits sharing the same apsidal line. For two coplanar elliptic orbits with different apsidal lines, the numerical solution [16] and the closed-form solution [17, 18] have been obtained for the cotangent transfer problem. In addition, Zhang et al. solved the two-impulse cotangent rendezvous problem between two coplanar elliptic orbits [19] and between elliptic and hyperbolic orbits [20], respectively. Different from two impulses are required for the rendezvous 
problem, a single impulse can fulfill the interception mission. Thus, the tangent-impulse interception problem requires the same TOF for two spacecraft and a transfer orbit tangent to the initial orbit. Then, for a given impulse point, the interception point is not arbitrary and needs to be solved.

This paper studies the coplanar tangent-impulse interception problem from an interceptor on elliptic parking orbits to a target on hyperbolic orbits. Based on four special initial true anomalies, the solution-existence ranges of the target true anomaly are obtained for different impulse points. Then the one-tangent-impulse interception trajectories are obtained by solving a single-variable piecewise function.

\section{Problem Statement}

Assume that the interceptor moves on the initial elliptic parking orbit and the target moves on the target hyperbolic orbit, and the initial and target orbits are coplanar with different apsidal lines. At the initial time, the interceptor is at $P_{10}$ and the target is at $P_{20}$ (see Figure 1). Let the motion direction of both orbits be counterclockwise. The coasting arc for the interceptor is from $P_{10}$ to $P_{1}$, and the initial coasting time before the tangent impulse is $t_{1}$. The coasting arc for the target is from $P_{20}$ to $P_{2}$, and the coasting time of the target is $t_{2}$. The tangent impulse indicates that the transfer orbit is tangent to the initial elliptic orbit at $P_{1}$, and the transfer time is $t_{3}$. For the orbital interception problem, the flight time of the interceptor and that of the target are the same. For convenience, let

$$
\eta \triangleq \frac{1}{T_{1}}\left(t_{2}-t_{1}-t_{3}\right),
$$

where $t_{j}(j=1,2,3)$ denotes the coasting time with zero revolution and $T_{j}(j=1,3)$ denotes the orbital period, where the subscripts "1", " 2 ," and " 3 " denote the initial, target, and transfer orbits, respectively. For the upper-bounded impulse interception, the minimum-time solution is interesting; then the revolution number for the transfer orbit is set to be 0 . Then the orbital interception problem with a tangent impulse is to solve the impulse point $P_{1}$ and the interception point $P_{2}$ such that the following expression is satisfied:

$$
\eta \in \mathcal{N}=\{0,1,2, \ldots\}
$$

in which $\eta$ is a positive integer indicates that the interceptor moves multiple revolutions before the first tangent impulse on point $P_{1}$.

For the two-impulse cotangent rendezvous problem, if the final true anomaly $f_{2}$ is given, there is a single closedform solution for the initial true anomaly $f_{1}$; then $\eta$ in (1) is a function only of $f_{2}$ for zero revolution. However, for the one-tangent-impulse interception problem, from the above analysis, it is known that $\eta$ is a function of two variables, the initial true anomaly $f_{1}$ of the impulse point,and the target true anomaly $f_{2}$ of the interception point. Usually, there is an upper-bound constraint on the fuel consumption $\Delta v$; then the interception problem is to solve all values of $f_{1}$ and $f_{2}$ such that (2) is satisfied and the fuel consumption is less than a given value. In this paper, firstly the interception problem will

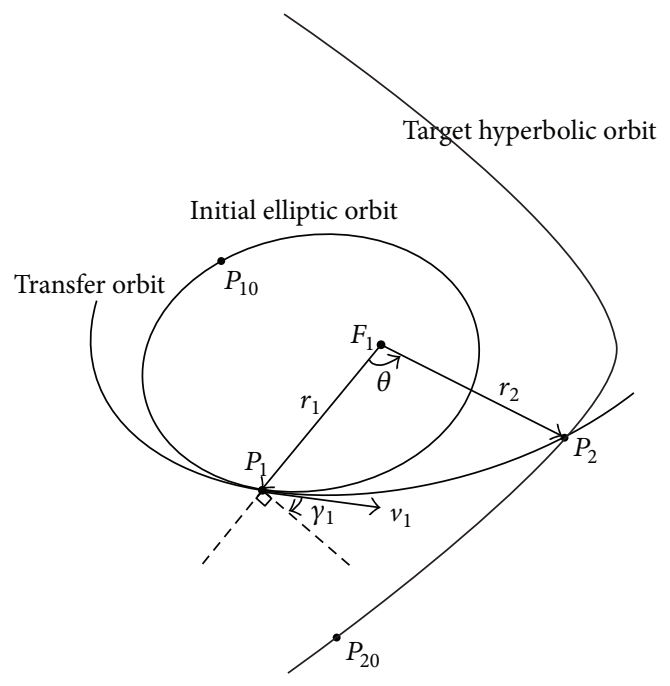

FIGURE 1: Geometrical interpretation of tangent-impulse interception for a hyperbolic target.

be solved at a given $f_{1}$ of the impulse point; then all feasible solutions will be obtained at different $f_{1}$ within one initial orbital period.

\section{Time-of-Flight Equations}

3.1. Coasting Time for the Initial/Target Orbit. For the initial elliptic orbit, the coasting time from $P_{10}$ to $P_{1}$ can be obtained by Kepler's equation. For the target hyperbolic orbit, the coasting time from $P_{20}$ to $P_{2}$ is [16]

$$
\begin{aligned}
t_{2}= & \frac{r_{20} r_{2} \sin \left(f_{2}-f_{20}\right)}{\sqrt{\mu p_{2}}} \\
& +\sqrt{\frac{-a_{2}^{3}}{\mu}}\left[\sinh \left(H_{2}-H_{20}\right)-\left(H_{2}-H_{20}\right)\right] \\
= & r_{20} \sqrt{\frac{p_{2}}{\mu}} \frac{\sin \left(f_{2}-f_{20}\right)}{1+e_{2} \cos f_{2}} \\
& +\sqrt{\frac{-a_{2}^{3}}{\mu}}\left[\sinh \left(H_{2}-H_{20}\right)-\left(H_{2}-H_{20}\right)\right],
\end{aligned}
$$

where $\mu$ is the gravitational parameter, $r$ is the radius of $P, f$ is the true anomaly, $p=a\left(1-e^{2}\right)$ is the semilatus rectum, $a$ is the semimajor axis, $e$ is the eccentricity, and $H$ is the hyperbolic anomaly, which is related to the true anomaly by

$$
\begin{aligned}
& \sinh H_{2}=\frac{\sqrt{e_{2}^{2}-1} \sin f_{2}}{1+e_{2} \cos f_{2}} \\
& \cosh H_{2}=\frac{e_{2}+\cos f_{2}}{1+e_{2} \cos f_{2}}
\end{aligned}
$$


from which it is known that

$$
\begin{aligned}
H_{2} & =\log \left(\sinh H_{2}+\cosh H_{2}\right) \\
& =\log \left(\frac{\sqrt{e_{2}^{2}-1} \sin f_{2}+e_{2}+\cos f_{2}}{1+e_{2} \cos f_{2}}\right) .
\end{aligned}
$$

Then the derivative of $\mathrm{H}_{2}$ with respect to $f_{2}$ is

$$
\frac{\mathrm{d} H_{2}}{\mathrm{~d} f_{2}}=\frac{\sqrt{e_{2}^{2}-1}}{1+e_{2} \cos f_{2}} .
$$

Furthermore, the derivative of $t_{2}$ with respect to $f_{2}$ is

$$
\begin{aligned}
\frac{\mathrm{d} t_{2}}{\mathrm{~d} f_{2}}= & r_{20} \sqrt{\frac{p_{2}}{\mu}} \frac{\cos \left(f_{2}-f_{20}\right)+e_{2} \cos f_{20}}{\left(1+e_{2} \cos f_{2}\right)^{2}} \\
& +\sqrt{\frac{-a_{2}^{3}}{\mu}}\left[\cosh \left(H_{2}-H_{20}\right)-1\right] \frac{\sqrt{e_{2}^{2}-1}}{1+e_{2} \cos f_{2}} .
\end{aligned}
$$

3.2. Transfer Time between the Initial and Target Orbits. The initial flight-path angle at $P_{1}$ can be written as [8]

$$
\gamma=\arctan \left(\frac{e_{1} \sin f_{1}}{1+e_{1} \cos f_{1}}\right) .
$$

Let the transfer angle be $\theta$, and [9]

$$
\begin{gathered}
\lambda=\frac{r_{2}(1-\cos \theta)}{r_{1} \cos ^{2} \gamma-r_{2} \cos (\theta+\gamma) \cos \gamma}, \\
v_{1 t}=\sqrt{\frac{\mu \lambda}{r_{1}}},
\end{gathered}
$$

where $v_{1 t}$ denotes the magnitude of the velocity vector at point $P_{1}$ on the transfer orbit. For different values of $\lambda$, there are three cases for the transfer time $[9,19]$.

(1) If $\lambda \in(0,2)$, the transfer trajectory is an elliptic orbit and the transfer time is

$$
\begin{aligned}
t_{3}= & \frac{2}{\sqrt{\mu}}\left(\frac{r_{1}}{2-\lambda}\right)^{3 / 2} \tan ^{-1}\left(\frac{\sqrt{2 / \lambda-1}}{\cos \gamma \cot (\theta / 2)-\sin \gamma}\right) \\
& +r_{2} \sqrt{\frac{r_{1}}{\mu \lambda}} \frac{\tan \gamma(1-\cos \theta)+(1-\lambda) \sin \theta}{(2-\lambda) \cos \gamma} .
\end{aligned}
$$

(2) If $\lambda>2$, the transfer trajectory is a hyperbolic orbit and the transfer time is

$$
\begin{aligned}
t_{3}= & r_{2} \sqrt{\frac{r_{1}}{\mu \lambda}} \\
& \times \frac{\tan \gamma(1-\cos \theta)+(1-\lambda) \sin \theta}{(2-\lambda) \cos \gamma} \\
& -\frac{1}{\sqrt{\mu}}\left(\frac{r_{1}}{\lambda-2}\right)^{3 / 2} \\
& \times \ln \frac{\sin \gamma-\cos \gamma \cot (\theta / 2)-(1-2 / \lambda)^{1 / 2}}{\sin \gamma-\cos \gamma \cot (\theta / 2)+(1-2 / \lambda)^{1 / 2}} .
\end{aligned}
$$

(3) If $\lambda=2$, the transfer trajectory is a parabolic orbit and the transfer time is

$$
\begin{aligned}
& t_{3}=\frac{2}{3} \sqrt{\frac{r_{1}^{3}}{\mu \lambda}} {\left[\frac{3 \cos \gamma \cot (\theta / 2)}{[\cos \gamma \cot (\theta / 2)-\sin \gamma]^{2}}\right.} \\
&\left.+\frac{1}{[\cos \gamma \cot (\theta / 2)-\sin \gamma]^{3}}\right] .
\end{aligned}
$$

It should be notified that ((11)-(13)) are functions independent of $v_{1 t}$.

The derivative of the transfer time $t_{3}$ with respect to the true anomaly $f_{2}$ is

$$
\frac{\mathrm{d} t_{3}}{\mathrm{~d} f_{2}}=\left(\frac{\partial t_{3}}{\partial r_{2}}+\frac{\partial t_{3}}{\partial \lambda} \frac{\partial \lambda}{\partial r_{2}}\right) \frac{\mathrm{d} r_{2}}{\mathrm{~d} f_{2}}+\left(\frac{\partial t_{3}}{\partial \theta}+\frac{\partial t_{3}}{\partial \lambda} \frac{\partial \lambda}{\partial \theta}\right) \frac{\mathrm{d} \theta}{\mathrm{d} f_{2}},
$$

where the partial derivatives $\partial t_{3} / \partial r_{2}, \partial t_{3} / \partial \lambda, \partial t_{3} / \partial \theta, \partial \lambda / \partial r_{2}$, and $\partial \lambda / \partial \theta$ and the full derivative $\mathrm{d} r_{2} / \mathrm{d} f_{2}$ are obtained in $[19,20]$ and $\mathrm{d} \theta / \mathrm{d} f_{2}=1$. The partial derivatives $\partial t_{3} / \partial \lambda, \partial t_{3} / \partial \theta$ are different for elliptic transfers and hyperbolic transfers, but the partial derivatives $\partial t_{3} / \partial r_{2}, \partial \lambda / \partial r_{2}$, and $\partial \lambda / \partial \theta$ and the full derivative $\mathrm{d} r_{2} / \mathrm{d} f_{2}$ are the same for elliptic orbits and hyperbolic orbits. Note that the target true anomalies $f_{2}$ for parabolic orbits are not continuous; thus the expression $\mathrm{d} t_{3} / \mathrm{d} f_{2}$ is not derived for $\lambda=2$.

For a given impulse point, the initial coasting time $t_{1}$ for the interceptor is independent of $f_{2}$. Then the derivative of $\eta$ with respect to $f_{2}$ is

$$
\eta^{\prime} \triangleq \frac{\mathrm{d} \eta}{\mathrm{d} f_{2}}=\frac{1}{T_{1}}\left(\frac{\mathrm{d} t_{2}}{\mathrm{~d} f_{2}}-\frac{\mathrm{d} t_{3}}{\mathrm{~d} f_{2}}\right) .
$$

\section{Conditions of Solution Existence}

The above section gives the transfer time for any conic orbit. A necessary condition is $\lambda>0$. Since the initial flight-path angle $\gamma \in(-\pi / 2, \pi / 2)$, the initial flight-direction angle is

$$
\bar{\gamma}=\frac{\pi}{2}-\gamma
$$

For the coplanar orbits, the transfer angle is

$$
\theta=f_{2}+\chi, \quad \text { where } \chi \triangleq \omega_{2}-\omega_{1}-f_{1},
$$

where $\omega$ is the argument of perigee.

Let

$$
\begin{aligned}
& c_{1}=\sin (\chi-\bar{\gamma})+e_{2} \frac{r_{1}}{p_{2}} \sin \bar{\gamma}, \\
& c_{2}=\cos (\chi-\bar{\gamma}), \\
& c_{3}=-\frac{r_{1}}{p_{2}} \sin \bar{\gamma},
\end{aligned}
$$

where $p$ is the semilatus rectum. There are three cases for $\lambda>0$ [18]: (1) if $c_{3} / \sqrt{c_{1}^{2}+c_{2}^{2}}<-1, f_{2} \in[0,2 \pi)$; (2) if 
$c_{3} / \sqrt{c_{1}^{2}+c_{2}^{2}}>1$, there is no solution for $f_{2} ;(3)$ if $-1 \leq$ $c_{3} / \sqrt{c_{1}^{2}+c_{2}^{2}} \leq 1$, then the range of $f_{2}$ for $\lambda>0$ is

$$
\begin{aligned}
& \arcsin \left(\frac{c_{3}}{\sqrt{c_{1}^{2}+c_{2}^{2}}}\right)-\operatorname{atan} 2\left(c_{1}, c_{2}\right) \\
& <f_{2}<\pi-\arcsin \left(\frac{c_{3}}{\sqrt{c_{1}^{2}+c_{2}^{2}}}\right)-\operatorname{atan} 2\left(c_{1}, c_{2}\right),
\end{aligned}
$$

where the four-quadrant inverse tangent function $\alpha=$ $\operatorname{atan} 2\left(b_{1}, b_{2}\right)$ is defined as the angle satisfying both $\sin \alpha=$ $b_{1} / \sqrt{b_{1}^{2}+b_{2}^{2}}$ and $\cos \alpha=b_{2} / \sqrt{b_{1}^{2}+b_{2}^{2}}$.

For any elliptical transfer orbit, $0<\lambda<2$ is required. Let

$$
\begin{aligned}
& c_{4}=\frac{p_{2}}{r_{1}} \cos (2 \bar{\gamma})+e_{2}(1-\cos (2 \gamma)) \cos \chi, \\
& c_{5}=\frac{p_{2}}{r_{1}} \sin (2 \bar{\gamma})+e_{2}(1-\cos (2 \gamma)) \sin \chi, \\
& c_{6}=\frac{p_{2}}{r_{1}}+\cos (2 \bar{\gamma})-1 .
\end{aligned}
$$

Then there are also three cases to be discussed [18]: (1) if $c_{6} / \sqrt{c_{4}^{2}+c_{5}^{2}}<-1, f_{2} \in[0,2 \pi)$; (2) if $c_{6} / \sqrt{c_{4}^{2}+c_{5}^{2}}>1$, there is no solution for $f_{2}$; (3) if $-1 \leq c_{6} / \sqrt{c_{4}^{2}+c_{5}^{2}} \leq 1$, then the range of $f_{2}$ for $0<\lambda<2$ can be expressed as

$$
\begin{aligned}
& -\chi-\operatorname{atan} 2\left(c_{4}, c_{5}\right)+\arcsin \left(\frac{c_{6}}{\sqrt{c_{4}^{2}+c_{5}^{2}}}\right) \\
& \quad<f_{2}<\pi-\chi-\operatorname{atan} 2\left(c_{4}, c_{5}\right)-\arcsin \left(\frac{c_{6}}{\sqrt{c_{4}^{2}+c_{5}^{2}}}\right) .
\end{aligned}
$$

In addition, the range of $f_{1}$ for existing elliptic transfers needs to be solved. If there exists some range of $f_{2}$, for $0<$ $\lambda<2$, the following inequality must be satisfied:

$$
c_{4}^{2}+c_{5}^{2}-c_{6}^{2} \geq 0
$$

Substituting (20) into (22) yields

$$
\begin{aligned}
& {[1-\cos (2 \bar{\gamma})]} \\
& \quad \times\left\{\left(e_{2}^{2}-1\right)[1-\cos (2 \bar{\gamma})]+2 \frac{p_{2}}{p_{1}}\left(1+e_{1} \cos f_{1}\right)\right. \\
& \left.\quad \times\left[1+e_{2} \cos (2 \bar{\gamma}-\chi)\right]\right\} \geq 0
\end{aligned}
$$

Note that the flight-direction angle $\bar{\gamma} \in(0, \pi / 2)$; then $\cos (2 \bar{\gamma})<1$ is satisfied for any $f_{1}$; thus (23) can be rewritten as

$$
\begin{aligned}
F \triangleq & \left(e_{2}^{2}-1\right)[1-\cos (2 \bar{\gamma})] \\
& +2 \frac{p_{2}}{p_{1}}\left(1+e_{1} \cos f_{1}\right)\left[1+e_{2} \cos (2 \bar{\gamma}-\chi)\right] \geq 0 .
\end{aligned}
$$

From ((16), (17)) it is known that $\bar{\gamma}$ and $\chi$ are functions only of $f_{1}$, then $F$ is a function only of $f_{1}$. The values of $f_{1}$ for the equality $F=0$ can be obtained by numerical methods; finally the range for inequality $F>0$ in (24) is obtained.

\section{Special Initial True Anomalies and the Solution-Existence Range}

There are four special points on the initial orbit, whose directions of velocity vectors are parallel to either of the lines of asymptotes for the target hyperbolic orbit. These initial true anomalies $f_{1, s j}(j=1,2,3,4)$ are solved in the following paragraphs. Let $u_{s j}=f_{1, s j}+\omega_{1}, \quad(j=1,2,3,4), u_{2 \max }=$ $f_{2 \max }+\omega_{2}=\arccos \left(-1 / e_{2}\right)+\omega_{2}$, and $u_{2 \min }=\arccos \left(1 / e_{2}\right)+$ $\omega_{2}$. From the geometry of Figure 2(a) it is known that

$$
\begin{aligned}
u_{s 1}+\bar{\gamma}_{s 1} & =u_{2 \max } \Longrightarrow f_{1, s 1}+\omega_{1}+\bar{\gamma}_{s 1} \\
& =\arccos \left(-\frac{1}{e_{2}}\right)+\omega_{2} .
\end{aligned}
$$

Note that the flight-direction angle is a function only of the true anomaly; then the above equation can be rewritten as

$$
\begin{aligned}
f_{1, s 1}+ & \omega_{1}+\frac{\pi}{2}-\arctan \left(\frac{e_{1} \sin f_{1, s 1}}{1+e_{1} \cos f_{1, s 1}}\right) \\
& =\arccos \left(-\frac{1}{e_{2}}\right)+\omega_{2}
\end{aligned}
$$

which is a function only of $f_{1, s 1}$. Thus, the classic NewtonRaphson iterative algorithm can be used to obtain the numerical solution for (26).

From the geometry of Figure 2(b), it is known that

$$
f_{1, s 2}+\omega_{1}+\bar{\gamma}_{s 2}-\left[\arccos \left(\frac{1}{e_{2}}\right)+\omega_{2}\right]=\pi
$$

which can be rewritten in a function only of $f_{1, s 2}$ as

$$
\begin{aligned}
f_{1, s 2} & +\omega_{1}-\frac{\pi}{2}-\arctan \left(\frac{e_{1} \sin f_{1, s 2}}{1+e_{1} \cos f_{1, s 2}}\right) \\
& =\arccos \left(\frac{1}{e_{2}}\right)+\omega_{2} .
\end{aligned}
$$

Similarly, from the geometries of Figures 2(c) and 2(d), it is known that the function only of $f_{1, s 3}$ is

$$
\begin{aligned}
f_{1, s 3} & +\omega_{1}-\frac{\pi}{2}-\arctan \left(\frac{e_{1} \sin f_{1, s 3}}{1+e_{1} \cos f_{1, s 3}}\right) \\
& =\arccos \left(-\frac{1}{e_{2}}\right)+\omega_{2}
\end{aligned}
$$




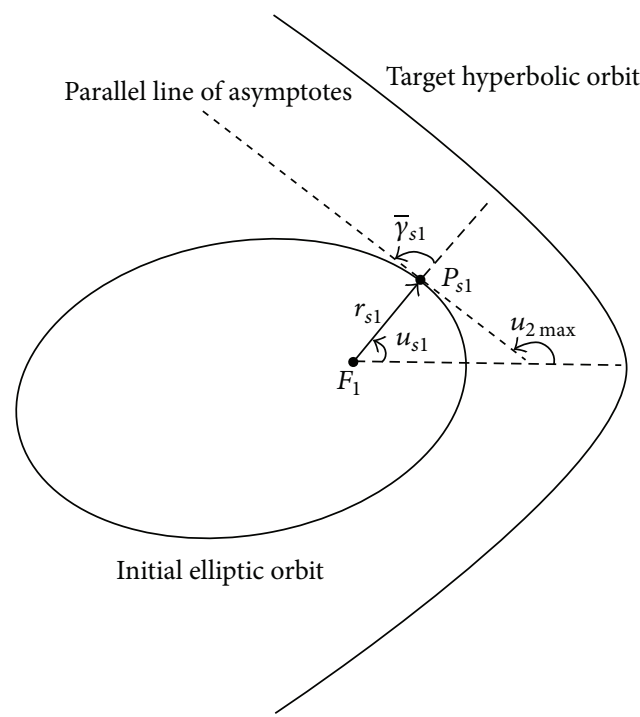

(a) $f_{1, s 1}$

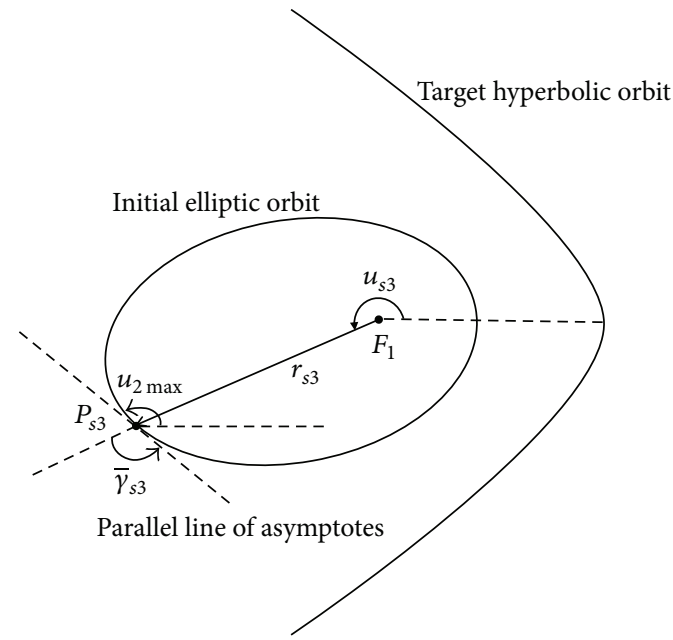

(c) $f_{1, s 3}$

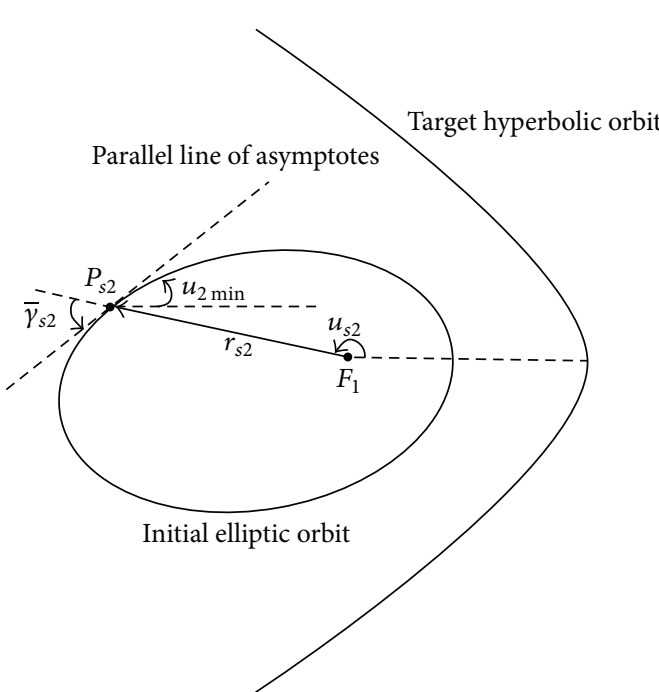

(b) $f_{1, s 2}$

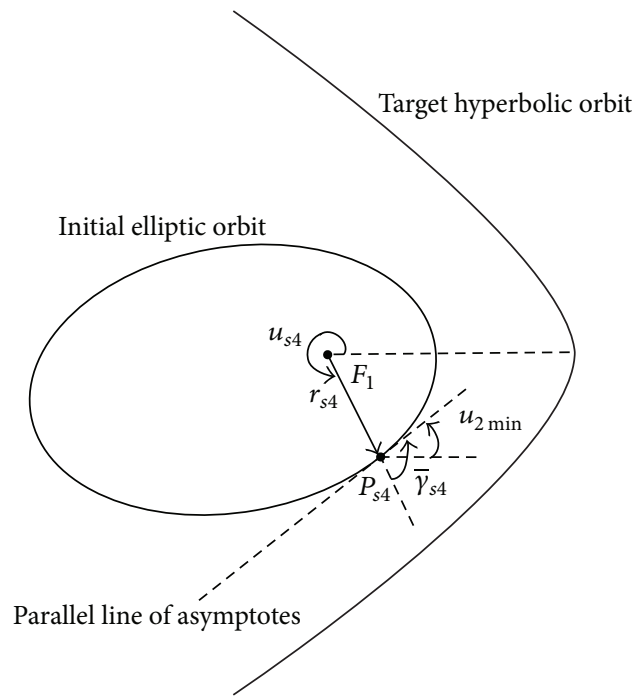

(d) $f_{1, s 4}$

FIGURE 2: Geometry interpretation for special initial true anomalies.

and the function only of $f_{1, s 4}$ is

$$
\begin{aligned}
f_{1, s 4}+ & \omega_{1}-\frac{3 \pi}{2}-\arctan \left(\frac{e_{1} \sin f_{1, s 4}}{1+e_{1} \cos f_{1, s 4}}\right) \\
& =\arccos \left(\frac{1}{e_{2}}\right)+\omega_{2} .
\end{aligned}
$$

All the values of $f_{1, s j},(j=1,2,3,4)$ are obtained by NewtonRaphson iterative algorithm.

Once the special initial true anomalies are obtained, the solution-existence range of target true anomaly $f_{2}$ can be discussed and obtained as follows.

(1) If $f_{1} \in\left[f_{1, s 1}, f_{1, s 2}\right]$, there is only one intersection point for the velocity-vector line and the target hyperbolic orbit. Assume that the true anomaly of the intersection point on the target hyperbolic orbit is $f_{2 q}$, which can be obtained by (19); then the target true anomaly is in the range $\left(-f_{2 \max }, f_{2 q}\right)$. The solution-existence range for any conic transfer orbit is obtained as follows. (a) If there is no range for elliptic transfer orbits, there is also no range for any conic transfer orbit. (b) If there exists a range $\left(f_{2 a}, f_{2 b}\right) \subset\left(-f_{2 \max }, f_{2 q}\right)$ for elliptic transfer orbits, which is obtained by (21), then the range for any conic transfer orbit can be $\left(f_{2 a}, f_{2 b}\right)$ or $\left(-f_{2 \max }, f_{2 b}\right)$. When $t_{3}$ by (11)-(13) is positive for an arbitrarily selected $f_{2} \in\left(-f_{2 \max }, f_{2 a}\right)$, for example, $f_{2}=\left(-f_{2 \max }+\right.$ $\left.f_{2 a}\right) / 2$, the solution-existence range is $\left(-f_{2 \max }, f_{2 b}\right)$; otherwise, the range is $\left(f_{2 a}, f_{2 b}\right)$.

(2) If $f_{1} \in\left(f_{1, s 2}, f_{1, s 3}\right)$, there are two intersection points for the velocity-vector line and the target 
hyperbolic orbit. Assume that the true anomalies of the intersection points on the target hyperbolic orbit are $f_{2 p}$ and $f_{2 q}$; then the solution-existence range for any conic transfer orbit is in the range $\left(f_{2 p}, f_{2 q}\right)$. The solution-existence range for elliptic transfer orbits is obtained as $\left(f_{2 a}, f_{2 b}\right) \subset\left(f_{2 p}, f_{2 q}\right)$ by (21). Then the solution-existence range for any conic transfer orbit is $\left(f_{2 p}, f_{2 b}\right)$.

(3) If $f_{1} \in\left[f_{1, s 3}, f_{1, s 4}\right]$, there is only one intersection point for the velocity-vector line and the target hyperbolic orbit. Assume that the true anomaly of the intersection point on the target hyperbolic orbit is $f_{2 p}$, which is obtained by (19); then the target true anomaly is in the range $\left(f_{2 p}, f_{2 \max }\right)$. The solutionexistence range for any conic transfer orbit is obtained as follows. (a) If there is no range for elliptic transfer orbits, then the solution-existence range for any conic transfer orbit is $\left(f_{2 p}, f_{2 \text { max }}\right)$. (b) If there exists a range $\left(f_{2 a}, f_{2 b}\right) \subset\left(f_{2 p}, f_{2 \max }\right)$, for elliptic transfer orbits, then the range for any conic transfer orbit can be $\left(f_{2 p}, f_{2 b}\right)$ or $\left(f_{2 p}, f_{2 \text { max }}\right)$. When $t_{3}$ by $((11)-(13))$ is positive for an arbitrarily selected $f_{2} \in\left(f_{2 b}, f_{2 \max }\right)$, for example, $f_{2}=\left(f_{2 b}+f_{2 \max }\right) / 2$, the solutionexistence range is $\left(f_{2 p}, f_{2 \text { max }}\right)$; otherwise, the range is $\left(f_{2 p}, f_{2 b}\right)$.

(4) If $f_{1} \in\left(f_{1, s 4}, f_{1, s 1}\right)$, there are two intersection points for the velocity-vector line and the target hyperbolic orbit. Assume that the true anomalies of the intersection points on the target hyperbolic orbit are $f_{2 p}$ and $f_{2 q}$; then the target true anomaly must be in the range $\left(f_{2 q}, f_{2 \text { max }}\right)$. There is no range for elliptic transfer orbits, and the solution-existence range for any conic (hyperbolic) transfer orbit is $\left(f_{2 q}, f_{2 \max }\right)$.

From the above discussions it is known that there may be no solution-existence range for any conic transfer orbit only when $f_{1} \in\left[f_{1, s 1}, f_{1, s 2}\right]$, and there is certainly no solution-existence range for elliptic transfer orbits when $f_{1} \in$ $\left[f_{1, s 4}, f_{1, s 1}\right]$.

Let the solution-existence range for any conic transfer by the above method be $\left(f_{2 m}, f_{2 n}\right)$. The target true anomaly $f_{2 i}$ at the impulse time is obtained by the hyperbolic orbit propagation. Then there are three cases. (1) If $f_{2 i}<f_{2 m}$, then the final solution should be obtained in the range $\left(f_{2 m}, f_{2 n}\right)$. (2) If $f_{2 i} \in\left[f_{2 m}, f_{2 n}\right]$, then the final solution should be obtained in the range $\left(f_{2 i}, f_{2 n}\right)$. (3) If $f_{2 i}>f_{2 n}$, then there is no solution.

\section{Solving the Problem}

For a given impulse point, the solution-existence range for any conic transfer and that for elliptic transfers are obtained in Section 5. Assume that the final solution-existence range for any conic transfer considering the target true anomaly at the impulse time is $\left(f_{2, g 1}, f_{2, g_{2}}\right)$. The interception problem can be solved by numerical methods.

The value of $\eta^{\prime}$ can be calculated by (15); then the solutions of $f_{2}$ for $\eta^{\prime}\left(f_{2}\right)=0$ can be obtained by a numerical iterative algorithm, for example, the golden section search. If there is no solution for $\eta^{\prime}\left(f_{2}\right)=0$, then $\eta$ decreases or increases monotonically. Then for the range $\left(f_{2, g 1}, f_{2, g_{2}}\right)$, if $\left\lfloor\eta\left(f_{g 1}+\right.\right.$ $\delta)\rfloor=\left\lfloor\eta\left(f_{g 2}-\delta\right)\right\rfloor$ or $\max \left(\left\lfloor\eta\left(f_{g 1}+\delta\right)\right\rfloor,\left\lfloor\eta\left(f_{g 2}-\delta\right)\right\rfloor\right)<0$, where the function $\lfloor y\rfloor$ denotes the nearest integer less than or equal to $y$, and $\delta$ is a small positive number, then there is no solution for (2). Otherwise, there are two cases.

(1) If $\min \left(\left\lfloor\eta\left(f_{g 1}+\delta\right)\right\rfloor,\left\lfloor\eta\left(f_{g 2}-\delta\right)\right\rfloor\right) \geq 0$, there are $\left\lfloor\left\lfloor\eta\left(f_{g 2}-\delta\right)\right\rfloor-\left\lfloor\eta\left(f_{g 1}+\delta\right)\right\rfloor \mid\right.$ solutions, which can be obtained by the secant method as

$$
\begin{array}{r}
f_{2, n+2}=f_{2, n+1}-\left(f_{2, n+1}-f_{2, n}\right) \frac{\eta\left(f_{2, n+1}\right)-N_{1}}{\eta\left(f_{2, n+1}\right)-\eta\left(f_{2, n}\right)}, \\
n \in \mathcal{N},
\end{array}
$$

where the initial guesses are $f_{2,0}=f_{g 1}+\delta, f_{2,1}=f_{g 2}-$ $\delta, N_{1}=\left\lfloor\eta\left(f_{g 1}+\delta\right)\right\rfloor+1,\left\lfloor\eta\left(f_{g 1}+\delta\right)\right\rfloor+2, \ldots,\left\lfloor\eta\left(f_{g 2}-\delta\right)\right\rfloor$ if $\left\lfloor\eta\left(f_{g 1}+\delta\right)\right\rfloor<\left\lfloor\eta\left(f_{g 2}-\delta\right)\right\rfloor$ and $N_{1}=\left\lfloor\eta\left(f_{g 2}-\delta\right)\right\rfloor+1$, $\left\lfloor\eta\left(f_{g 2}-\delta\right)\right\rfloor+2, \ldots,\left\lfloor\eta\left(f_{g 1}+\delta\right)\right\rfloor$ if $\left\lfloor\eta\left(f_{g 1}+\delta\right)\right\rfloor>$ $\left\lfloor\eta\left(f_{g 2}-\delta\right)\right\rfloor$.

(2) If $\min \left(\left\lfloor\eta\left(f_{g 1}+\delta\right)\right\rfloor,\left\lfloor\eta\left(f_{g 2}-\delta\right)\right\rfloor\right)<0$, there are $\left[\max \left(\left\lfloor\eta\left(f_{g 1}+\delta\right)\right\rfloor,\left\lfloor\eta\left(f_{g 2}-\delta\right)\right\rfloor\right)+1\right]$ solutions, which can be obtained by (31) with $N_{1}=0,1, \ldots$, $\max \left(\left\lfloor\eta\left(f_{g 1}+\delta\right)\right\rfloor,\left\lfloor\eta\left(f_{g 2}-\delta\right)\right\rfloor\right)$. Note that $\eta\left(f_{g 1}\right)$ instead of $\eta\left(f_{g 1}+\delta\right)$ should be used for the range $\left[f_{g 1}, f_{g 2}\right)$.

If there exist $k_{n} \geq 1$ solutions for $\eta^{\prime}\left(f_{2}\right)=0$ and these $k_{n}$ extreme points for $\eta\left(f_{2}\right)$ are obtained as $f_{2, k 1}<f_{2, k 2}<$ $\cdots<f_{2, k n}$, then there are $k_{n}+1$ piecewise ranges, that is, $\left(f_{2, g 1}, f_{2, k 1}\right),\left[f_{2, k 1}, f_{2, k 2}\right), \ldots,\left[f_{2, k n}, f_{2, g_{2}}\right)$. For each piecewise range, the function of $\eta$ decreases or increases monotonically. Then the solution for each piecewise range can be obtained by (31) with different initial guesses. If $\lim _{\left(f_{2} \rightarrow f_{2,92}\right)} \eta=+\infty$ (see Figure $3(\mathrm{~d})$ ), there are infinite solutions in the range $\left[f_{2, k n}, f_{2, g_{2}}\right.$ ), and the minimum-time solution (or with the minimum $f_{2}$ ) will be obtained.

In the patched-conic approximation method, the radius of the Earth's sphere of influence is $R_{\mathrm{SOI}}=925,000 \mathrm{~km}$; then the true anomaly range of the solution is $f_{2} \in\left(-f_{\mathrm{SOI}}, f_{\mathrm{SOI}}\right)$, where $f_{\text {SOI }}=\arccos \left(\left[p_{2} / R_{\text {SOI }}-1\right] / e_{2}\right)$. Thus, the solutions beyond this range should be removed.

The above section gives a method to obtain all solutions for a given initial impulse point $f_{1}$. For each solution, the corresponding fuel consumption is

$$
\begin{aligned}
\Delta v & =\left|v_{1 t}-v_{1}\right| \\
& =\left|\sqrt{\frac{\mu}{p_{1}} \lambda\left(1+e_{1} \cos f_{1}\right)}-\sqrt{\frac{\mu}{p_{1}}\left(1+e_{1}^{2}+2 e_{1} \cos f_{1}\right)}\right| .
\end{aligned}
$$

The solution whose cost is greater than the given maximal value should be removed. Thus, the feasible solution needs to satisfy two conditions, including the radius of the Earth's sphere of influence and the given maximal value of fuel. When considering the initial coasting time before 


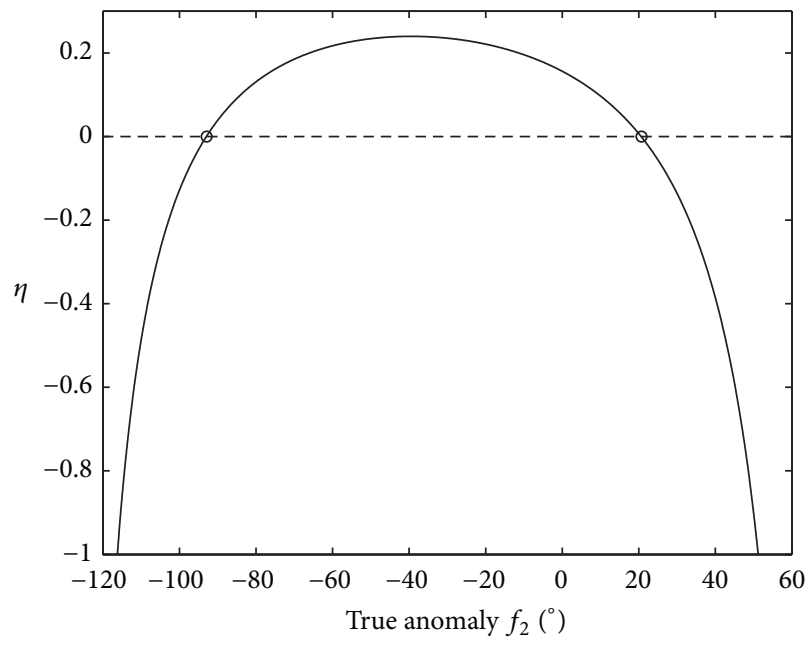

(a) $f_{1}=160^{\circ}$

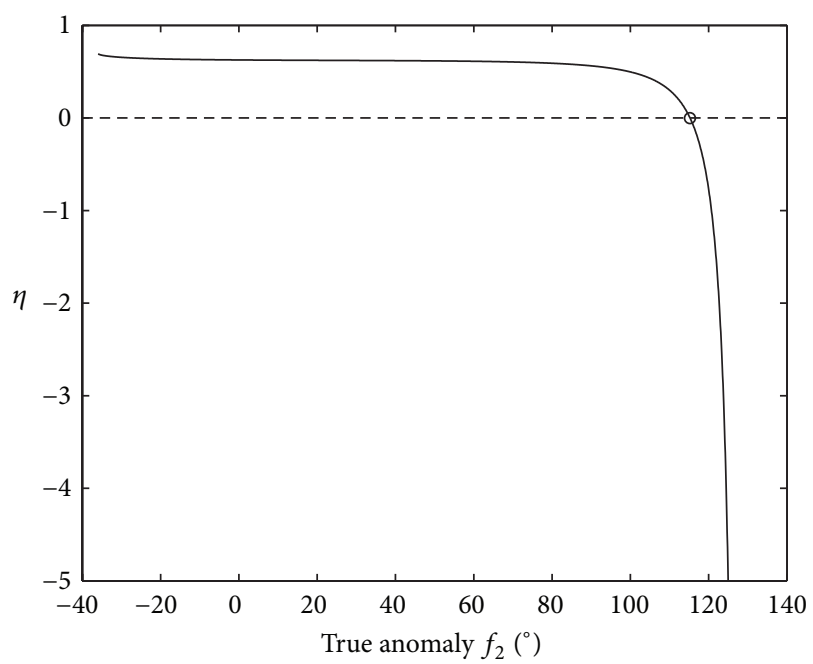

(c) $f_{1}=240^{\circ}$

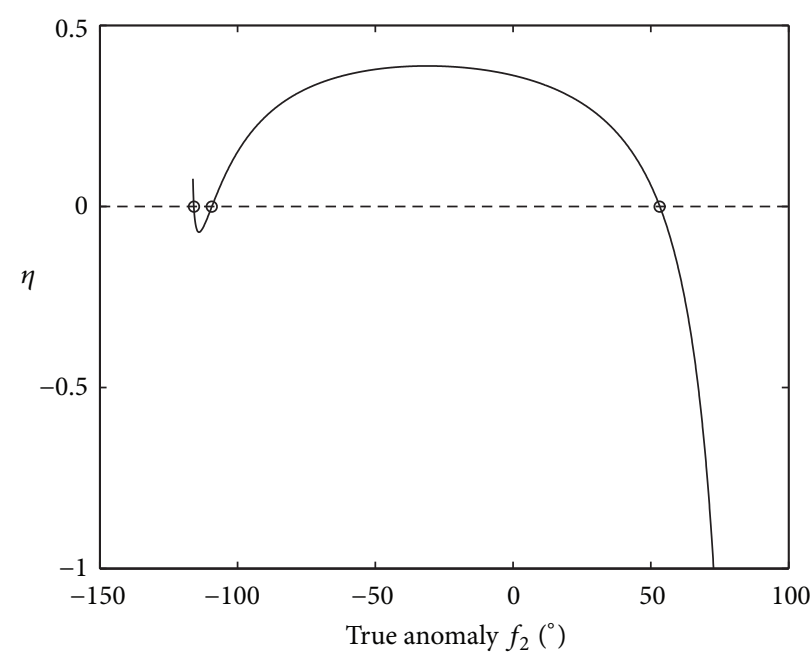

(b) $f_{1}=170^{\circ}$

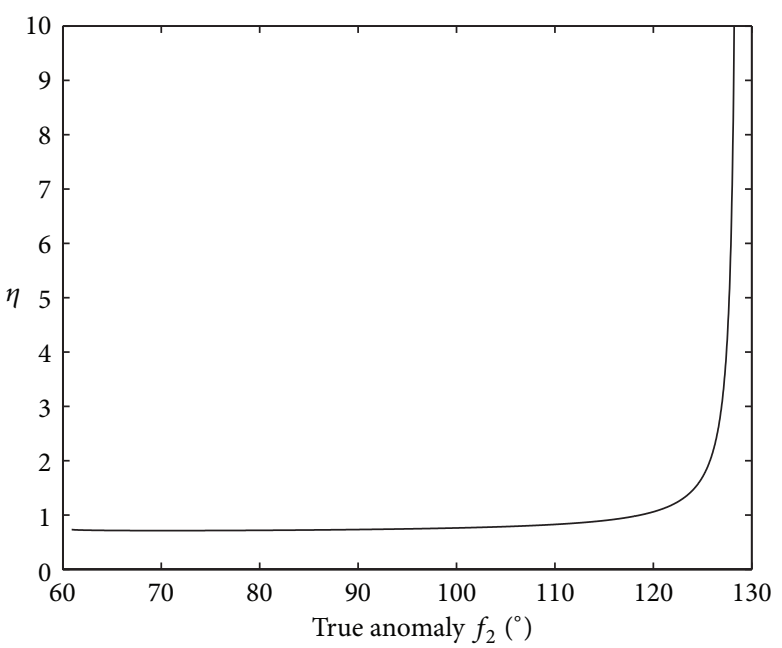

(d) $f_{1}=330^{\circ}$

Figure 3: The curves of $\eta$ versus $f_{2}$ for different $f_{1}$.

the impulse, the impulse point is free, then for each initial impulse point, all feasible solutions can be obtained and the minimum-time one is with the minimum value of $f_{2}$.

Reference [20] dealt with the cotangent rendezvous between two coplanar elliptic and hyperbolic orbits (Problem 1 ), whereas this paper is for the tangent-impulse interception problem from an interceptor on elliptic parking orbits to a target on hyperbolic orbits (Problem 2). Three main differences between these two problems are listed as follows.

(1) For Problem 1, there is a single closed-form solution for an arbitrary point on the target hyperbolic orbit; that is, $f_{2} \in\left(-f_{2 \max }, f_{2 \max }\right)$, where $f_{2 \max }=$ $\arccos \left(-1 / e_{2}\right)$; thus there is only one degree of freedom in (2) for zero revolution; however, in a specified (e.g., counterclockwise) motion direction, the solution-existence range is not the whole range of target true anomaly. For Problem 2, there is no certain relationship between the tangent-impulse point $f_{1}$ and the interception point $f_{2}$. In other words, there are two degrees of freedom in (2) with zero revolutions.

(2) For Problem 1, with nonintersecting case (counterclockwise), the solution-existence range for cotangent rendezvous is $f_{2} \in\left(f_{2 a}, f_{2 \max }\right)$, where $\left(f_{2 a}, f_{2 b}\right)$ is obtained by the solution-existence range for elliptic transfers [20]. But for Problem 2 with nonintersecting case (counterclockwise), the solution-existence range of $f_{2}$ can be obtained by the proposed method for different $f_{1}$, which is dependent on four special initial true anomalies whose velocity vectors are parallel to the lines of asymptotes for the target hyperbolic orbit. Moreover, there may be no solution-existence range for any conic transfer orbit when $f_{1} \in\left[f_{1, s 1}, f_{1, s 2}\right]$.

(3) For Problem 1, with a given revolution number $N_{3}$ of the transfer orbit, usually there is only one orbit solution (see the examples in [20]). However, for 
Problem 2, with an upper-bounded impulse, the minimum-time solution is interesting; then $N_{3}$ is set to be 0 . For a given impulse point $f_{1}$, there may exist multiple solutions of $f_{2}$ (see Figures 3(a) and 3(b)). With the initial coasting time, all feasible solutions for different impulse points are obtained and the minimum-time one is with the minimum value of $f_{2}$.

\section{Numerical Examples}

Assume that the interceptor's orbit elements are $a_{1}=2 R_{E}+$ $4,000 \mathrm{~km}, e_{1}=0.6, \omega_{1}=10^{\circ}$, and $f_{10}=60^{\circ}$ and the target's orbit elements are $a_{2}=-2 R_{E}-10,000 \mathrm{~km}, e_{2}=1.6, \omega_{2}=0^{\circ}$, and $f_{20}=-120^{\circ}$, where $R_{E}=6378.13 \mathrm{~km}$ is the Earth's radius. The gravitational parameter used for the example is $\mu=398,600.4415 \mathrm{~km}^{3} / \mathrm{s}^{2}$. The upper bound of the tangent impulse is set to be $5 \mathrm{~km} / \mathrm{s}$. Four special initial true anomalies are obtained as $f_{1, s 1}=45.4187^{\circ}, f_{1, s 2}=158.1023^{\circ}, f_{1, s 3}=$ $191.9457^{\circ}$, and $f_{1, s 4}=284.5334^{\circ}$ via (26), (28), (29), and (30). To obtain elliptic transfers, the range of the initial true anomaly of the impulse point is $f_{1} \in\left(79.9244^{\circ}, 249.9268^{\circ}\right)$ via (24). In addition, considering the radius of the Earth's sphere of influence, that is, $925,000 \mathrm{~km}$, the range of true anomaly is $f_{2} \in\left(-126.9425^{\circ}, 126.9425^{\circ}\right)$.

If the impulse is imposed at the initial time, that is, $f_{1}=$ $f_{10}=60^{\circ}$, then $f_{1} \in\left[f_{1, s 1}, f_{1, s 2}\right]$ is satisfied, and there is no range for elliptic transfers; thus no solution-existence range exists for $f_{2}$.

If the impulse is imposed at $f_{1}=160^{\circ}$, the target true anomaly at the impulse time is $f_{2}=-118.3547^{\circ}$ by solving Kepler's equation for hyperbolic orbits, and the plots of $\eta$ versus $f_{2}$ can be obtained in Figure 3(a). The final solution-existence range for any conic transfer considering the target true anomaly at the impulse time is $\left(f_{2, g 1}, f_{2, g_{2}}\right)=\left(-118.3547^{\circ}, 72.4802^{\circ}\right)$. There are two solutions, that is, $-92.8589^{\circ}$ and $20.7296^{\circ}$; the corresponding revolution numbers are both 0 and the corresponding costs are $1.3182 \mathrm{~km} / \mathrm{s}$ and $1.0211 \mathrm{~km} / \mathrm{s}$, respectively. The minimumtime solution is $-92.8589^{\circ}$, the corresponding transfer time is $21972.4 \mathrm{~s}$, and the total interception time $t_{2}$ is $28310.4 \mathrm{~s}$ via (3). The value of $\lambda$ is obtained as 1.1178 by (9); thus the trajectory is elliptic and it is plotted in Figure 4.

If the impulse is imposed at $f_{1}=170^{\circ}$, the target true anomaly at the impulse time is $f_{2}=-117.7828^{\circ}$, the plots of $\eta$ versus $f_{2}$ can be obtained in Figure $3(\mathrm{~b})$. The final solutionexistence range is $\left(f_{2, g 1}, f_{2, g 2}\right)=\left(-116.3047^{\circ}, 88.3112^{\circ}\right)$. There are three solutions, $-115.8579^{\circ},-109.2932^{\circ}$, and $53.2432^{\circ}$, all the corresponding revolution numbers are 0 , and the corresponding costs are $21.1998 \mathrm{~km} / \mathrm{s}, 3.9088 \mathrm{~km} / \mathrm{s}$, and $1.1770 \mathrm{~km} / \mathrm{s}$, respectively. The energy cost of $-115.8579^{\circ}$ is much greater than $5 \mathrm{~km} / \mathrm{s}$; thus it cannot be used. The feasible minimum-time solution is $-109.2932^{\circ}$, the corresponding transfer time is $13188.3 \mathrm{~s}$, and the total interception time is 21258.8 s. The value of $\lambda$ is 2.7636 ; thus the trajectory is hyperbolic and it is plotted in Figure 5.

If the impulse is imposed at $f_{1}=240^{\circ}$, the target true anomaly at the impulse time is $f_{2}=-112.5985^{\circ}$; the plots of $\eta$ versus $f_{2}$ can be obtained in Figure 3(c). There is a single

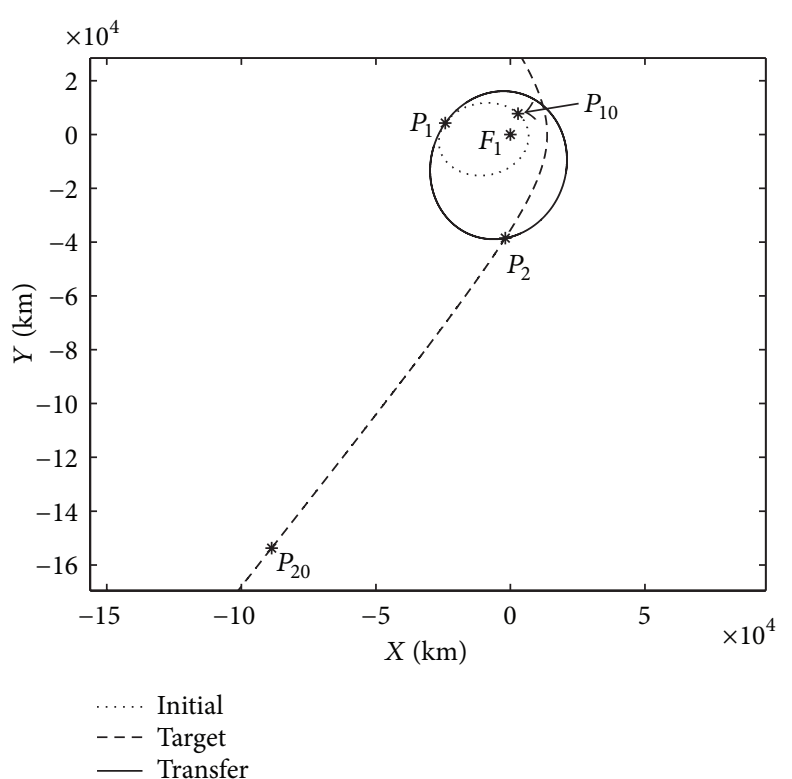

FIGURE 4: Tangent-impulse interception with elliptic transfer trajectory.

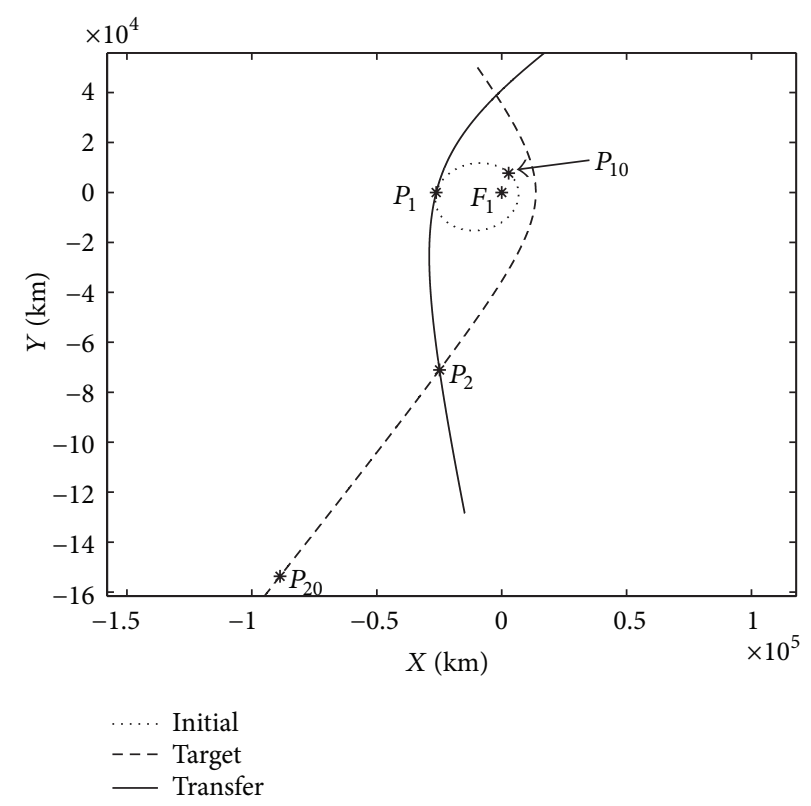

FIGURE 5: Tangent-impulse interception with hyperbolic transfer trajectory.

solution $115.2336^{\circ}$, the corresponding revolution number is 0 , the cost is $1.7743 \mathrm{~km} / \mathrm{s}$, the transfer time is $36318.2 \mathrm{~s}$, and the total interception time is $54208.1 \mathrm{~s}$. The value of $\lambda$ is 1.9316 ; thus the trajectory is elliptic.

If the impulse is imposed at $f_{1}=330^{\circ}$, the target true anomaly at the impulse time is $f_{2}=-110.2890^{\circ}$, the plots of $\eta$ versus $f_{2}$ can be obtained in Figure $3(\mathrm{~d})$, and there are infinite solutions. However, when considering the sphere of influence of the Earth, there are three solutions. The minimum-time solution is $118.6911^{\circ}$, the corresponding revolution number 
TABLE 1: Feasible solutions of $f_{2}$ for different $f_{1}$ and the corresponding interception time $t_{2}$ and $\Delta v$.

\begin{tabular}{lccc}
\hline$f_{1}\left({ }^{\circ}\right)$ & $\begin{array}{c}\text { Solutions of } \\
\left(f_{2}\left({ }^{\circ}\right), N_{1}\right)\end{array}$ & $t_{2}(\mathrm{~s})$ & $\Delta v(\mathrm{~km} / \mathrm{s})$ \\
\hline 150 & $(-56.6897,0)$ & 32108.4 & 0.9035 \\
& $(-39.9134,0)$ & 32844.3 & 0.8897 \\
170 & $(-109.2932,0)$ & 21258.8 & 3.9088 \\
& $(53.2432,1)$ & 35834.5 & 1.1770 \\
190 & $(89.1630,0)$ & 39080.1 & 1.4596 \\
210 & $(104.3983,0)$ & 43665.1 & 1.6333 \\
230 & $(112.3591,0)$ & 49932.6 & 1.7338 \\
250 & $(117.7130,0)$ & 59847.2 & 1.8148 \\
270 & $(121.9959,0)$ & 80249.5 & 1.9082 \\
290 & $(125.9555,0)$ & 160241.3 & 2.0322 \\
310 & $(124.4383,1)$ & 111489.9 & 2.2976 \\
330 & $(118.6911,1)$ & 62895.4 & 2.9698 \\
& $(125.7512,2)$ & 150667.5 & 2.6872 \\
& $(126.9266,3)$ & 236873.8 & 2.6464 \\
350 & $(114.2511,1)$ & 52541.0 & 4.4692 \\
& $(124.2882,2)$ & 108532.2 & 3.5407 \\
& $(126.0145,3)$ & 163286.2 & 3.4192 \\
& $(126.7542,4)$ & 217544.3 & 3.3698 \\
& $(126.1114,4)$ & 168589.6 & 4.9766 \\
& $(126.6609,5)$ & 208476.8 & 4.8911 \\
\hline
\end{tabular}

is 1 , the corresponding costs is $2.9698 \mathrm{~km} / \mathrm{s}$, the corresponding transfer time is $20927.9 \mathrm{~s}$, and the total interception time is $62895.4 \mathrm{~s}$. The value of $\lambda$ is 2.7280 ; thus the trajectory is hyperbolic.

For different impulse points, all feasible solutions satisfying $f_{2} \in\left(-126.9425^{\circ}, 126.9425^{\circ}\right)$ and the fuel constraint and the corresponding value of $N_{2}=\eta$ for (2) can be obtained by (31) and are listed in Table 1 . The total interception time is $t_{2}=t_{1}+t_{3}$; the corresponding costs are also listed in Table 1 . It should be notified that there is no feasible solution when $f_{1}=30^{\circ}+k_{j} \cdot 20^{\circ}, k_{j}=1,2,3,4,5$. By using the numerical optimization algorithm, the minimum-time impulse point is at $f_{1}=170.5235^{\circ}$, the solution is $f_{2}=-110.8795^{\circ}$, the cost is $4.9999 \mathrm{~km} / \mathrm{s}$, and the total interception time is $19810.6 \mathrm{~s}$. The minimum-time interception trajectory is hyperbolic and it is near to that in Figure 5.

\section{Conclusions}

This paper studies the interception problem for a hyperbolic target with an upper-bounded tangent impulse. Based on four special initial true anomalies, the solution-existence range of the target true anomaly for any conic transfer is obtained. Then the problem is solved by the secant method for a time-of-flight equation. For a given impulse point, there may be zero, one, many, or infinite solutions; however, when considering the sphere of influence of the Earth, there are always finite solutions. For different impulse points, all feasible solutions and the global minimum-time solution are obtained. This proposed method is valid for an assigned impulse point and the time optimization with the initial coasting time. The interception with a tangent-impulse technique can be used in orbital interception task for potentially hazardous objects during the Earth flyby with simple control.

\section{Conflict of Interests}

The authors declare that there is no conflict of interests regarding the publication of this paper.

\section{Acknowledgments}

This work is supported in part by the China Postdoctoral Science Foundation Funded Project (Grant no. 2012M520753), the Heilongjiang Postdoctoral Science Foundation (Grant no. LBH-Z12125), and the National Natural Scientific Foundation of China (Grant no. 61273096).

\section{References}

[1] J. C. Solem, "Interception of comets and asteroids on collision course with Earth," Journal of Spacecraft and Rockets, vol. 30, no. 2, pp. 222-228, 1993.

[2] I. M. Ross, S.-Y. Park, and S. D. V. Porter, "Gravitational effects of earth in optimizing $\Delta \mathrm{V}$ for deflecting earth-crossing asteroids," Journal of Spacecraft and Rockets, vol. 38, no. 5, pp. 759-764, 2001.

[3] B. Dachwald and B. Wie, "Solar sail trajectory optimization for intercepting, impacting, and deflecting near-earth asteroids," in Proceedings of the AIAA Guidance, Navigation, and Control Conference and Exhibit, pp. 3331-3348, San Francisco, Calif, USA, August 2005.

[4] D. Izzo, "Optimization of interplanetary trajectories for impulsive and continuous asteroid deflection," Journal of Guidance, Control, and Dynamics, vol. 30, no. 2, pp. 401-408, 2007.

[5] J. Park, R. G. Rhinehart, and P. T. Kabamba, "Miss analysis in Lambert interceptions with application to a new guidance law," Mathematical Problems in Engineering: Theory, Methods and Applications, vol. 6, no. 2-3, pp. 225-265, 2000.

[6] Y. Ulybyshev, "Direct high-speed interception: analytic solutions, qualitative analysis, and applications," Journal of Spacecraft and Rockets, vol. 38, no. 3, pp. 351-359, 2001.

[7] S. P. Burns and J. J. Scherock, "Lambert guidance routine designed to match position and velocity of ballistic target," Journal of Guidance, Control, and Dynamics, vol. 27, no. 6, pp. 989-996, 2004.

[8] R. H. Battin, An Introduction to the Mathematics and Methods of Astrodynamics, American Institute of Aeronautics and Astronautics (AIAA), Reston, Va, USA, Revised edition, 1999.

[9] S. L. Nelson and P. Zarchan, "Alternative approach to the solution of Lambert's problem," Journal of Guidance, Control, and Dynamics, vol. 15, no. 4, pp. 1003-1009, 1992.

[10] P. V. Arlulkar and S. D. Naik, "Solution based on dynamical approach for multiple-revolution lambert problem," Journal of Guidance, Control, and Dynamics, vol. 34, no. 3, pp. 920-923, 2011.

[11] G. Zhang, D. Mortari, and D. Zhou, "Constrained multiplerevolution Lambert's problem," Journal of Guidance, Control, and Dynamics, vol. 33, no. 6, pp. 1779-1786, 2010. 
[12] C. A. Lembeck and J. E. Prussing, "Optimal impulsive intercept with low-thrust rendezvous return," Journal of Guidance, Control, and Dynamics, vol. 16, no. 3, pp. 426-433, 1993.

[13] D.-R. Taur, V. Coverstone-Carroll, and J. E. Prussing, "Optimal impulsive time-fixed orbital rendezvous and interception with path constraints," Journal of Guidance, Control, and Dynamics, vol. 18, no. 1, pp. 54-60, 1995.

[14] H. Leeghim, "Spacecraft intercept using minimum control energy and wait time," Celestial Mechanics \& Dynamical Astronomy, vol. 115, no. 1, pp. 1-19, 2013.

[15] D. A. Vallado, Fundamentals of Astrodynamics and Applications, Microcosm Press, El Segundo, Calif, USA, 2nd edition, 2001.

[16] B. F. Thompson, K. K. Choi, S. W. Piggott, and S. R. Beaver, "Orbital targeting based on hodograph theory for improved rendezvous safety," Journal of Guidance, Control, and Dynamics, vol. 33, no. 5, pp. 1566-1576, 2010.

[17] V. G. Adamyan, L. V. Adamyan, G. M. Zaimtsyan, and L. T. Manandyan, "Double-pulse cotangential transfers between coplanar elliptic orbits," Journal of Applied Mathematics and Mechanics, vol. 73, no. 6, pp. 664-672, 2009.

[18] G. Zhang, D. Zhou, D. Mortari, and T. A. Henderson, "Analytical study of tangent orbit and conditions for its solution existence," Journal of Guidance, Control, and Dynamics, vol. 35, no. 1, pp. 186-194, 2012.

[19] G. Zhang, D. Zhou, Z. Sun, and X. Cao, "Optimal two-impulse cotangent rendezvous between coplanar elliptical orbits," Journal of Guidance, Control, and Dynamics, vol. 36, no. 3, pp. 677685, 2013.

[20] G. Zhang, X. Cao, and D. Zhou, "Two-impulse cotangent rendezvous between coplanar elliptic and hyperbolic orbits," Journal of Guidance, Control, and Dynamics, vol. 37, no. 3, pp. 964969, 2014. 


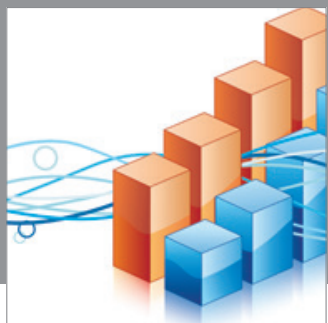

Advances in

Operations Research

mansans

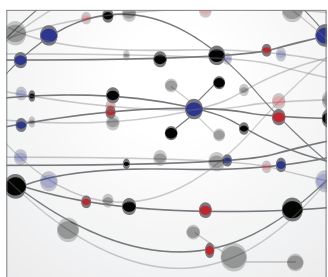

The Scientific World Journal
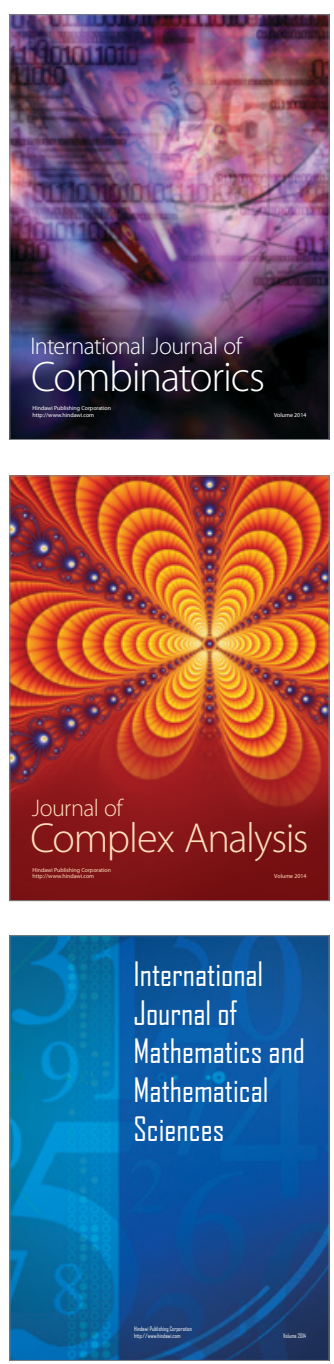
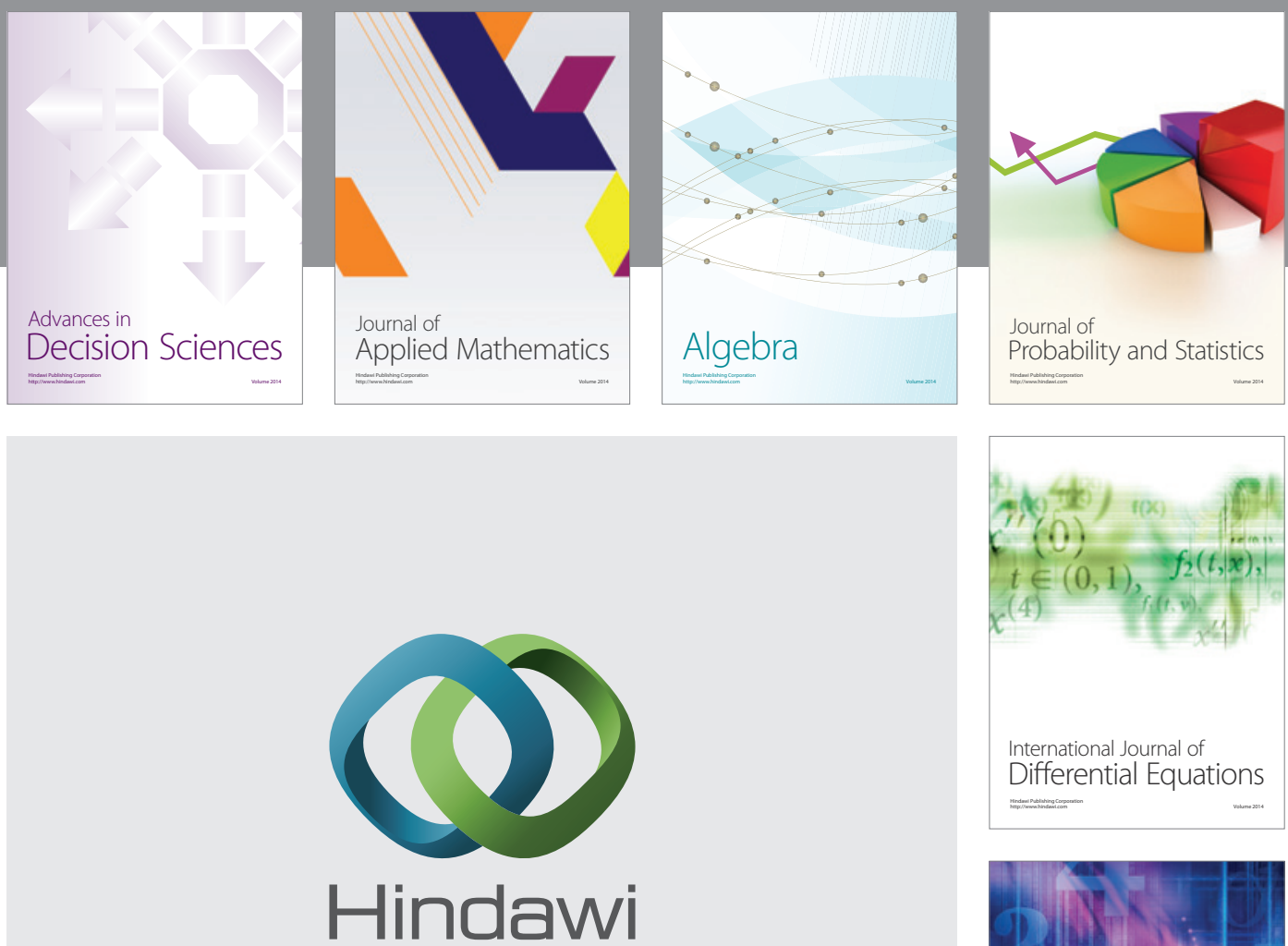

Submit your manuscripts at http://www.hindawi.com
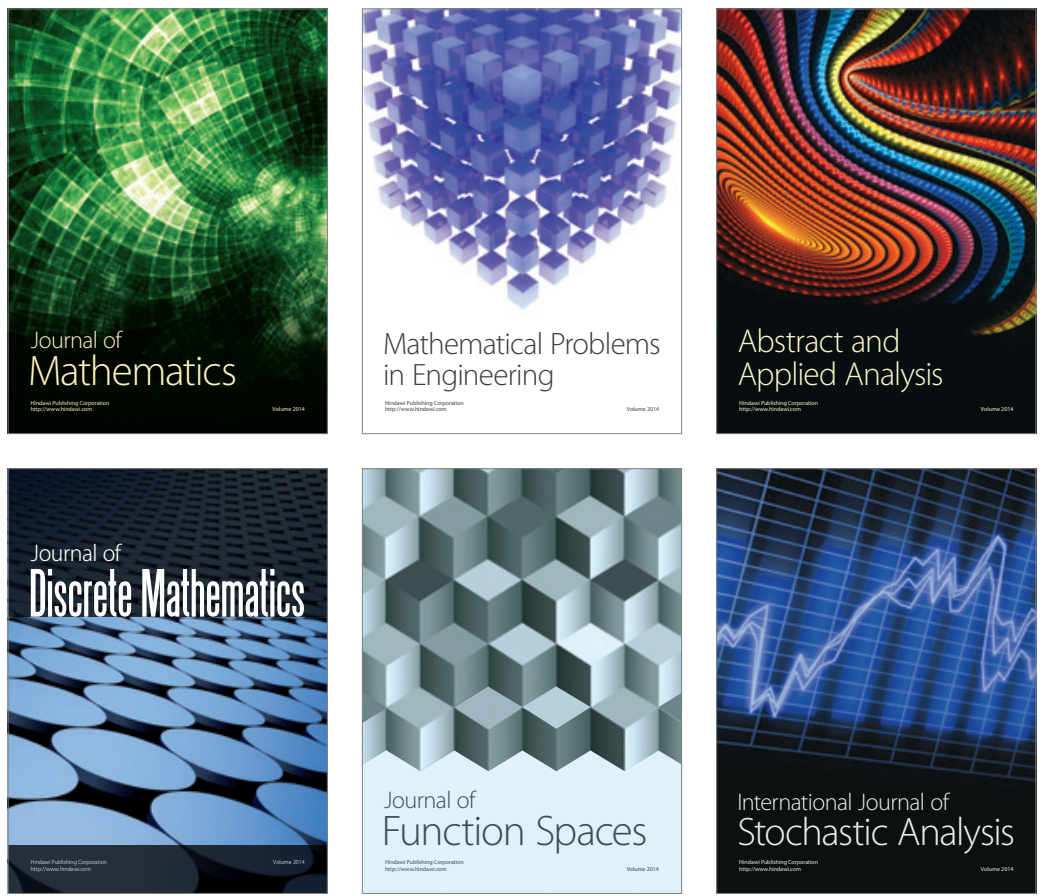

Journal of

Function Spaces

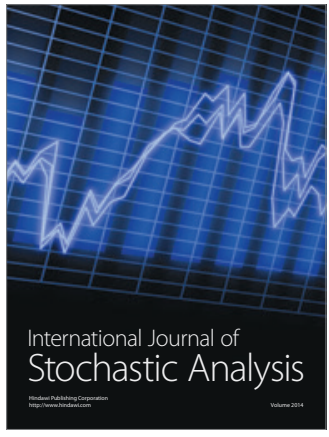

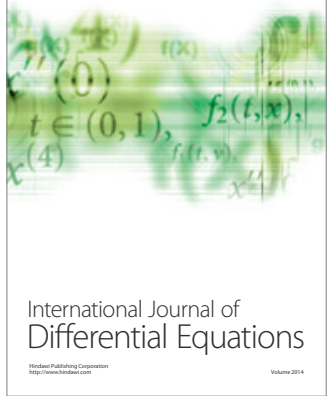
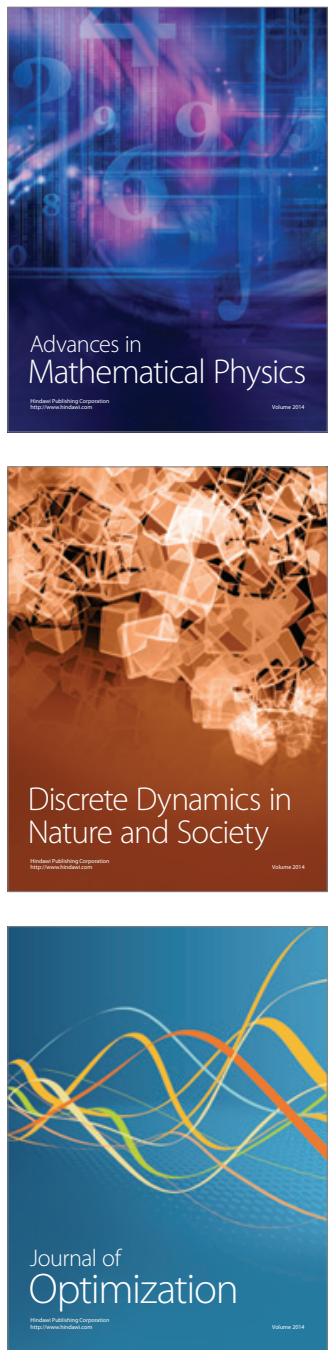